\title{
Leveraging Social Media Sources to Generate Personalized Music Playlists
}

\author{
Cataldo Musto, Giovanni Semeraro, Pasquale Lops, Marco de Gemmis, \\ Fedelucio Narducci \\ Department of Computer Science \\ University of Bari Aldo Moro, Italy \\ \{cataldomusto, semeraro, lops, degemmis, narducci\}@di.uniba.it
}

\begin{abstract}
This paper presents MyMusic, a system that exploits social media sources for generating personalized music playlists. This work is based on the idea that information extracted from social networks, such as Facebook and Last.fm, might be effectively exploited for personalization tasks. Indeed, information related to music preferences of users can be easily gathered from social platforms and used to define a model of user interests. The use of social media is a very cheap and effective way to overcome the classical cold start problem of recommender systems. In this work we enriched social media-based playlists with new artists related to those the user already likes. Specifically, we compare two different enrichment techniques: the first leverages the knowledge stored on DBpedia, the structured version of Wikipedia, while the second is based on the content-based similarity between descriptions of artists. The final playlist is ranked and finally presented to the user that can listen to the songs and express her feedbacks. A prototype version of $\mathrm{MyMu}-$ sic was made available online in order to carry out a preliminary user study to evaluate the best enrichment strategy. The preliminary results encouraged keeping on this research.
\end{abstract}

Keywords: Music Recommendation, Social Media, Personalization, DBPedia

\section{Introduction}

The concept of Information Overload describes a state where the efficiency is jeopardized by the amount of available information [7]. This definition perfectly fits with the Web navigation scenario, where users are overwhelmed by the continuous flow of data. Hence, Information Filtering (IF) tools, such as Recommender Systems (RS) are more and more needed, since their main goal is to optimize the access to data sources and provide users just with the relevant information according to their preferences. It is common to refer to these systems as personalization systems [16].

Even if the recent evolution of the Web is universally considered as one of the causes of information overload [10], the Web 2.0 phenomenon further worsened the problem and changed the rules for personalization. Indeed, the recent spread 
of social networks and collaborative platforms makes physiologically impossible to follow the information flow in real-time. Recent studies showed that more than 20 hours of video are uploaded every minute to YouTube ${ }^{1}$. So, despite users spent $22 \%$ of their web navigation time on these platforms ${ }^{2}$, the current scenario made the problem of information overload today felt also for images, video and audio contents. Consequently, it is necessary to adapt IF tools and techniques in order to make them able to properly handle multimedia contents as well. However, it is not correct to consider Web 2.0 solely as a source of problems. The other side of the coin is that social networks can be a rich source of information useful to automatically infer user preferences, and this can be helpful to mitigate the cold start problem of RS.

The main contribution of this work is MyMusic, a system that leverages different social media sources for generating personalized music playlists. The filtering model behind MyMusic is based on the assumption that information about music preferences can be easily gathered from Facebook profiles. Next, playlists built using explicit Facebook preferences may be enriched with new artists somehow related to those the user already likes. We propose and compare two different enrichment techniques: the first leverages the knowledge stored on DBpedia, the structured version of Wikipedia, while the second is based on the content-based similarity between descriptions of artists. The final playlist is then ranked and finally presented to the user that can express her feedback. A prototype version of MyMusic was made available online and a preliminary user study to detect the best enrichment technique was performed.

The paper is organized as follows. Section 2 gives a general overview of the most relevant related work in the area of music recommendation. The architecture of the systems is sketched in Section 3, together with the approaches for building personalized music playlists. Finally, Section 4 presents the results of the preliminary experimental evaluation, while Section 5 contains the conclusions and the future directions of this research.

\section{Related Work}

The topic of music recommendation has been widely covered in literature, and a good overview of the state of the art is given in [3] by Oscar Celma.

In the music domain, the commonly used technique for providing recommendations is collaborative filtering, implemented in very well known services, such as MyStrands ${ }^{3}$, Last.fm ${ }^{4}$ or iTunes Genius. MyStrands is a great service for music discovery and recommendation, based on songs/artists uploaded either from iTunes playlists or added as favorites on the site. It allows the recommendations of similar songs, albums, and artists. Last.fm is another interesting and widely adopted music recommendation service, whose features are improved by

\footnotetext{
${ }^{1} \mathrm{http}: / / \mathrm{www} \cdot$ youtube.com/t/press_timeline

${ }^{2}$ http://blog.nielsen.com/nielsenwire/social/

${ }^{3}$ http://www.mystrands.com

${ }^{4}$ http://www.last.fm
} 
the Last.fm Scrobbler, focusing on the music already played to help users to discover more music. Finally, iTunes Genius is the recommender system implemented within iTunes. It sifts the iTunes library to find songs that might be combined together, in order to obtain compelling compilations, such as personal channels that broadcast music just for that specific user.

An early attempt to recommend music using collaborative filtering was done by Shardanand [21]. However, collaborative filtering methods are not generally able to tackle the problem of finding items within the long tail of music production, those for which the amount of taste data is limited. This limits the recommendation of novel and serendipitous items, that is a fundamental feature of music recommender systems.

The recent trend is thus to use content-based recommendation strategies, which analyze diverse sets of low-level features (e.g. harmony, rhythm, melody) [14], or high-level features (metadata or content-based data available in social media) [9] to provide recommendations. Unlike collaborative filtering, contentbased models can effectively tackle the problem of new or unpopular items, typical of real use cases, where the information flow is continuous and uncontrolled, with new artists and songs continuously published. The most noticeable system using (manual) content-based descriptions to recommend music is Pandora $^{5}$, with 100 million registered users, more than 900 thousands songs and 90 thousands artists in catalog. The main problem of the system is the scalability, because the music annotation process is entirely done manually.

Conversely, FOAFing the music $[5,6]$ is able to recommend, discover and explore music content, based on user profiling via Friend of a Friend (FOAF) ${ }^{6}$ descriptions, context-based information extracted from music related RSS feeds, and content-based descriptions automatically extracted from the audio itself. The use of FOAF and in general of Linked Data is investigated in [17], that reports a wide range of music-related data sources that have been interlinked within the Linking Open Data initiative. For example, the DBTune project $^{7}$ exports several datasets encompassing detailed editorial information, geolocalization of artists, social networking information amongst artists and listeners, listening habits, and content-based data in RDF format. Such interlinking provides an open social graph that can be queried and processed in an uniform way. Inspired by this work, we decided to use DBPedia as a Linked Data source for enriching initial music preferences of users with related artists and songs.

Recently, Bu et al. [2] followed the recent trend of harvesting information coming from social media for personalization tasks and proposed its application for music recommendation. In [12], Lamere investigated the use of textual contents, such as tags, as source for music recommendation as well, and Wang et al. [22] showed the usefulness of tags with respect to other content-based sources. Thus, we decided to evaluate an alternative way that exploits tags for enriching initial music preferences of users with related artists and songs.

\footnotetext{
${ }^{5}$ http://www.pandora.com

${ }^{6}$ http://www.foaf-project.org

7 http://dbtune.org
} 
Another important feature of music recommender systems is represented by the context, in order to adapt recommendations to different situations. This aspect is out of the scope of the present work, but there is a body of work related to the problem of context-aware music recommendation, taking into account different contextual variables, such as time, weather data, traffic condition or driver's mood for users when in a car $[13,18,1]$.

Finally, a complete picture of recent trends in the music recommendation area can be found in [4].

\section{MyMusic: personalized playlists generator}

The general architecture of MyMusic is depicted in Figure 1. The playlist generation is performed in different steps, each handled by a specific component. The process is directly triggered by the user, who invokes the PLAYLIST GENERATOR module. The set of her favourite artists is built by mapping her preferences gathered from the Facebook profile with a set of artists extracted from Last.fm. Given this preliminary set, the PLAYLIST ENRICHER adds new artists using different enrichment strategies. Finally, for each artist in that set, the most popular tracks are extracted and ranked. The final playlist is then built and shown to the target user who can express her feedback on the proposed tracks.

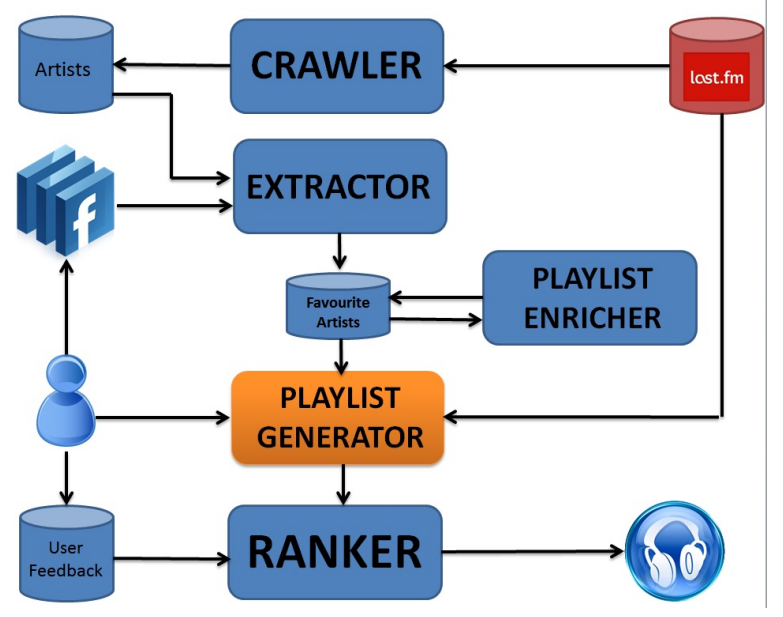

Fig. 1. MyMusic architecture

A working implementation of MyMusic is available online ${ }^{8}$, while a screenshot of the user interface is provided in Figure 2. There is a slideshow which allows the user to move among the tracks in the playlist and start listening some of

\footnotetext{
${ }^{8}$ http://193.204.187.223:8080/sssc
} 


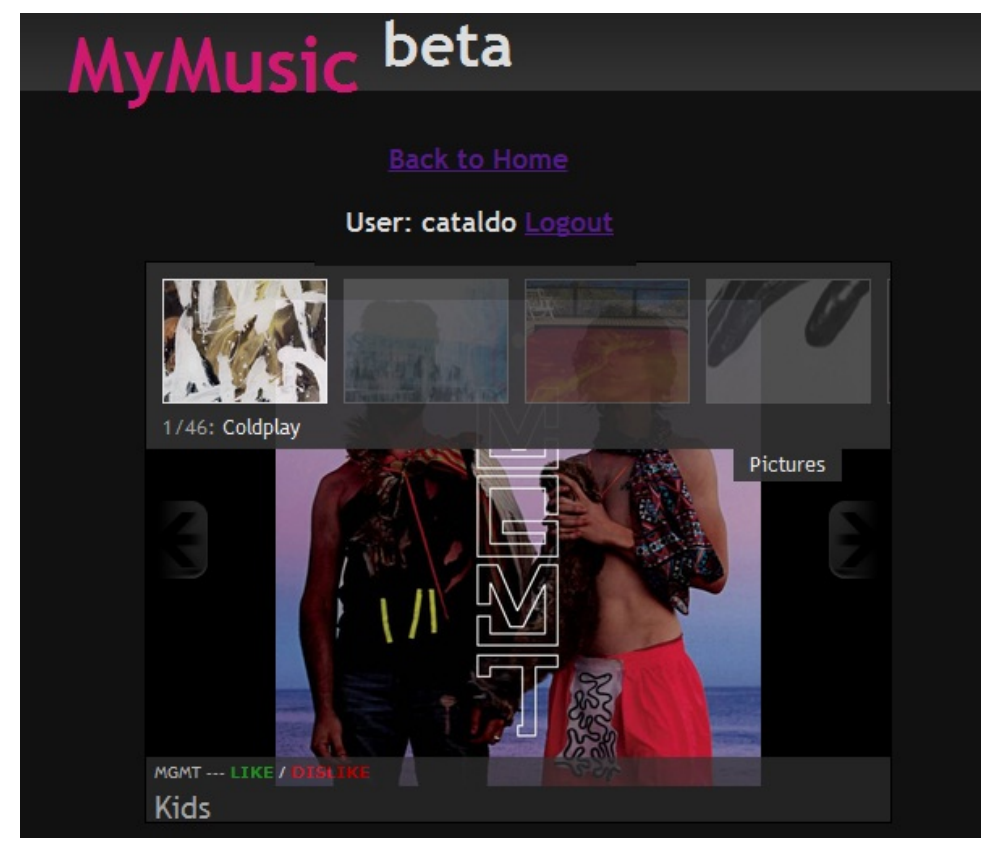

Fig. 2. MyMusic screenshot

them with a simple click. The lower part of the interface reports the title of the song, the name of the artist and the like/dislike button to allow users express a binary feedback.

More details about the components and the whole generation process follow.

Crawling Data from Last.fm MyMusic needs a set of artists to feed the playlist generation process. The CRAWLER module queries Last.fm through its public APIs in order to build a corpus of artists. This process is performed in a batch way, and it is scheduled in order to have an up to date set of data. For each artist in Last.fm, the name, a picture, the title of the most popular tracks, their playcount and a set of tags that describe that artist (see for example Figure 5) are crawled.

Extracting Preferences from Facebook A common weakness of personalization systems is the need of explicit preferences to learn profile of user interests in order to provide recommendations. Thanks to Web 2.0 applications, and specificically with the advent of social networks, gathering explicit preferences is becoming increasingly simple. Facebook profiles, for example, contain explicit information about the artists preferred by a user (Figure 3). The ExTRACTOR module of MyMusic connects to Facebook profiles, extracts user preferences related to music, and maps them with the data gathered from Last.fm in order 
to build a preliminary set of artists preferred by that user. Even if Facebook profiles also contain a lot of implicit data that might be analyzed to infer music preferences, such as links, attended events, content of posts, ..., the current version of MyMusic only relies on explicit data.

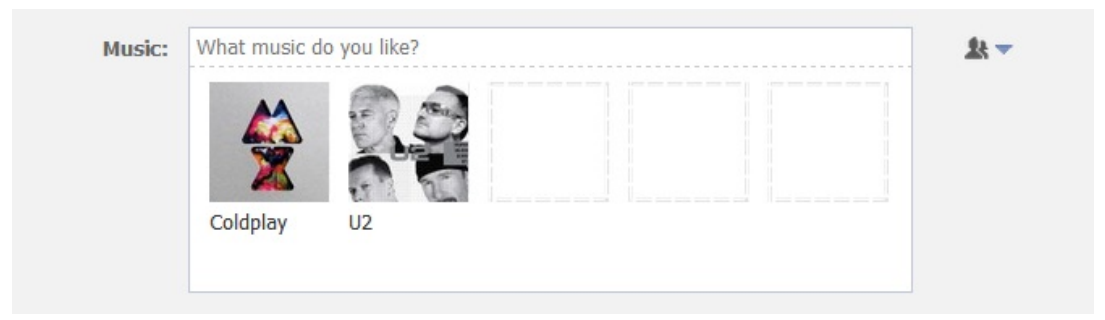

Fig. 3. User Preferences from a Facebook profile

Playlist Enrichment The main goal of the PLAYLIST ENRICHER module is to add new artists to the set of those preferred by the user and explicitly expressed in her Facebook profile. This should also help to discover new artists. Formally, given a user $u$, we define the set of favourite artists extracted from the Facebook profile as $F_{u}=\left\{f_{1}, f_{2}, \ldots, f_{n}\right\}$. For each $f_{i}$, the PLAYLIST ENRICHER builds a set $E_{f_{i}}=\left\{e_{1}, e_{2}, \ldots, e_{m}\right\}$ that contains the $m$ most similar artists among those stored in the corpus built using Last.fm. Finally, the final set of artists for user $u$ is defined as:

$$
E_{u}=\bigcup_{i=1}^{n} E_{f_{i}}
$$

In this work we have evaluated two different techniques for enriching the playlists with new artists. The first technique is based on the exploitation of DBPedia, while the second one uses the content-based similarity between artist representations.

Enrichment based on DBpedia. The first approach for enriching the playlist with new artists relies on the exploitation of $\mathrm{DBpedia}^{9}$, a project whose goal is to represent information stored in Wikipedia in a structured form by means of RDF triples [11]. Nowadays DBpedia represents the nucleus of the so-called Web of Data, since the information is made freely available online and it is possible to submit queries to get complex information by exploiting the structured nature of this data and the relationships among them. In DBpedia, each Wikipedia entry

\footnotetext{
${ }^{9}$ http://dbpedia.org
} 
is mapped in a DBpedia concept, and each concept is assigned with a unique Uniform Resource Identifier (URI), e.g. HTTP://DBPEDIA.oRG/RESOURCE/COLDPLAY, and each concept can be imagined as a node in a graph. Node pairs are connected by means of relations, called properties. As shown in Figure 4, thanks to DBpedia we can represent complex information in a structured form. For example, the following triple encodes the fact that Coldplay is a music band whose genre is Alternative Rock.

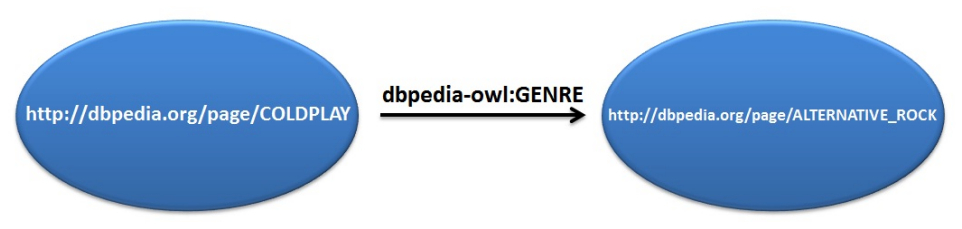

Fig. 4. An example of property in DBPedia

Our approach is based on the assumption that each artist can be mapped to a DBpedia node. The inceptive idea is that the similarity between two artists can be computed according to the number of properties they share (e.g. two Italian bands playing rock music are probably more similar than an Italian and an English band that play different genres). Consequently, we looked for properties that could be useful for computing similarity. We decided to use DBPEDIAOWL:GENRE (describing the genre of the artist) and DCTERMS:SUBJECT that provides information about the musical category of the artist. Operationally, we used a powerful query language, namely SPARQL - SPARQL Protocol and RDF Query Language - an RDF query language, similar to a query language for databases, able to retrieve and manipulate data stored in Resource Description Framework format. By simply querying a SPARQL endpoint containing information stored in DBpedia we can extract the set of artists related to those already liked by a user. For example, we extracted the set of artists related to Coldplay by using the following query:

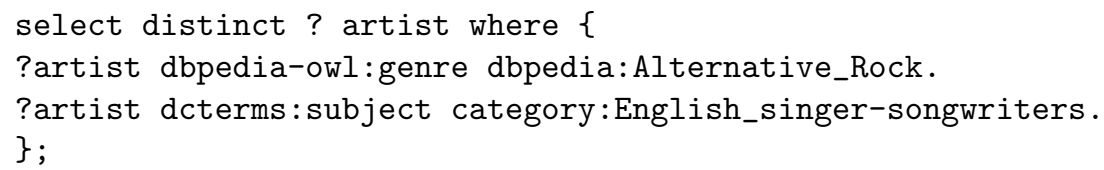

Since DBPedia returns an unordered set of results, returned artists are ranked according to their playcount in Last.fm. The first $m$ artists returned by the SPARQL endpoint are considered as relevant (and related) and added to the set of the favourite artists.

Enrichment based on a Content-based Model. As stated in Section 3, each artist in Last.fm is described through a set of tags as in classical collaborative 
tagging systems [8]. Each tag provides information about the genre played by the artist, such as rock, or about typical features of her songs, such as melanchonic (Figure 5). By following the classical Vector Space Model (VSM) [19], each artist can be represented as a point in a $n$-dimensional vector space, whose dimensions are all the $n$ different tags used to describe all the artists (vocabulary).

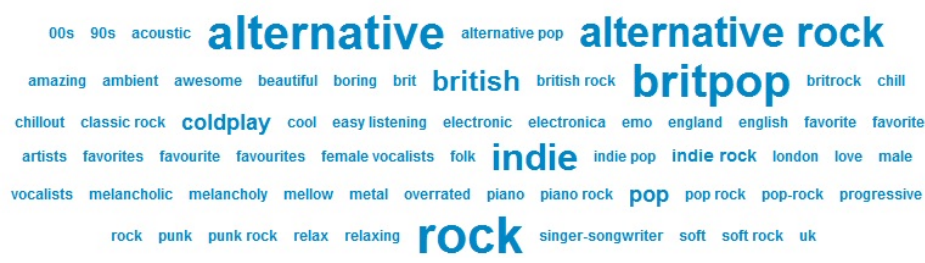

Fig. 5. Coldplay tag cloud from Last.fm

The rationale behind this enrichment strategy is that it might be easy to understand the similarity/relatedness of two artists by comparing tags used to describe them. Besides, we can suppose that artists most related to those the user already likes can be considered as relevant for her, as well. Thus, given a VSM-based representation, we can use cosine similarity to compute how much similar or related two artists are. More formally, given two artists $a$ and $b$, their correlation is computed as follows:

$$
\cos \operatorname{Sim}(a, b)=\frac{a \cdot b}{\|a\| \cdot\|b\|}=\frac{\sum_{i=1}^{n} a_{i} \times b_{i}}{\sqrt{\sum_{i=1}^{n}\left(a_{i}\right)^{2}} \times \sqrt{\sum_{i=1}^{n}\left(b_{i}\right)^{2}}}
$$

In this scenario, for each artist in $F_{u}$ (i.e. extracted from the Facebook profile) we compute the cosine similarity between the artist and all the others extracted from Last.fm. The $m$ artists with the highest cosine similarity are added by the PLAYLIST ENRICHER module to the list of favourite ones.

\subsection{Playlist Generation and Ranking}

The PLAYLIST GENERATOR module builds the set of candidate tracks by merging the songs played by the artists extracted from the Facebook profile with those played by artists added through the enrichment process. Finally, since the set of candidate tracks need to be ranked, the RANKER exploits a scoring function to sort the tracks in a descending relevance order. For each track in the candidate track list, the score is calculated as follows:

$$
\operatorname{score}\left(s_{i}\right)=\operatorname{play}\left(s_{i}\right) * \operatorname{source}\left(s_{i}\right)
$$

where $\operatorname{play}\left(s_{i}\right)$ is the normalized playcount of the song $s_{i}$ in the Last.fm community, while $\operatorname{source}\left(s_{i}\right)$ is a weight assigned to the source of the song. $\operatorname{source}\left(s_{i}\right)$ 
is set to $\alpha$ for tracks played by an artist in the Facebook profile, and $1-\alpha$ for tracks played by an artist produced by the enrichment process, in order to weigh differently explicit and implicit preferences.

\section{Experimental Evaluation}

The goal of the experimental evaluation is to identify the most effective enrichment technique able to generate the most relevant playlists for final users. We carried out the experiment by involving 30 users under 30 , heterogeneously distributed by sex and education, according to the availability sampling strategy. None of them performed studies in the music field. The final crawl of Last.fm was performed at the end of November, 2011, and data about 228,878 artists were extracted. For each artist we got also the top-5 tracks, so the final MyMusic dataset contained information about more than 1 million tracks. Each user explicitly granted the access to her Facebook profile to extract data about favourite artists. At the end of the extraction step 325 artists were extracted, so each user had about 10 favourite artists on average. Users left on the platform 462 feedbacks, almost 15 per user on average. For the enrichment technique based on DBpedia the SPARQL endpoint located at http://dbpedia.org was queried. It contained 3.64 million nodes and more than 100,000 are related to musical artists or albums. For the cosine similarity-based technique we exploited Last.fm APIs to extract the most popular tags associated to each artist. The less significant and meaningful tags (such as seenlive, cool, and so on) were considered as noisy and filtered out. In order to identify the best enrichment technique, we asked users to use the application for three weeks. In the first two weeks the system was set with a different enrichment technique, while in the last one a simple baseline based on the most popular artists on Last.fm was used, thus playlists were enriched with tracks played by the most popular artists regardless user preferences. For each round of the experimental evaluation the users granted the access to their Facebook preferences. Given the playlist generated by the system, we asked them to explicity express their feedback on the tracks played by the artists generated by the enrichment techniques. For each track it was possible to express a binary feedback (whether they liked the song or the don not) or to simply ignore the suggestion (See Figure 2). Users were not aware of the enrichment technique adopted in that round. Regarding the parameters, the value of $\alpha$ was set to 0.5 , so to favourite artists and to those added by the enrichment process was given an equal weight. This value was set through a rough heuristic. The parameter $m$ indicates the number of similar artists extracted for each favourite one, and we compared user behavior in three different configurations, with $m$ set to 1,2 and 3 . This means that we enriched each artist extracted from Facebook with respectively 1,2 or 3 new artists. The maximum size of the playlist was set to 50. To get the final results, we computed the precision of the system as the ratio between the number of positive feedbacks and the total number of suggested tracks. The overall results are reported in the Table 1. 
Table 1. Results of Experiments

\begin{tabular}{|c|c|c|c|}
\hline & \multicolumn{3}{|c|}{ Artists } \\
\hline Enrichment strategy & $\mathrm{m}=1$ & $\mathrm{~m}=2$ & $\mathrm{~m}=3$ \\
\hline DBpedia & $65.9 \%$ & $64.6 \%$ & $63.2 \%$ \\
\hline Tag-based similarity & $\mathbf{7 6 . 3 \%}$ & $\mathbf{7 5 . 2 \%}$ & $\mathbf{6 9 . 7 \%}$ \\
\hline Popularity & \multicolumn{3}{|c|}{$58 \%$} \\
\hline
\end{tabular}

It is worth to notice that the two enrichment strategies are able to outperform the baseline. This means that data extracted from social networks actually reflect user preferences and the intuition of modeling users according to the information gathered from social sources is valid. The enrichment technique that gained the best performance is that based on the tag-based similarity. It overcomes the DBpedia-based strategy of roughly 10 points with $m=1$ and $m=2$ and $6 \%$ with $m=3$, and the baseline between 11 and 18 points \%. Even though this technique gained the best results, a deeper analysis can provide different outcomes. Indeed, with $m=3$ the gap between DBpedia and tag-based similarity decreases of almost 5 points. This means that with higher values of $m$ a pure content-based representation introduces much more noise than DBpedia, whose effectiveness remains constant (only 2 points $\%$ of difference among the different configurations). In other terms, with low similarity values, it is likely that the enrichment technique based on tag-based similarity suggests artists not actually interesting for the target user. The introduction of some thresholding strategy might be useful to avoid this problem. The good results obtained by the baseline based on popularity can be justified by the low diversity of the users involved in the evaluation. Since most of them like very common artists, such as U2 and Coldplay, a simple popularity-based approach is very accurate. It is likely that, by involving users with a more specific musical knowledge and uncommon preferences, results obtained by the baseline may get worse. Despite its results, the DBpedia-based enrichment technique may represent a valuable alternative to avoid the typical drawbacks of pure content-based representations. Indeed, it might be helpful for providing explanations about the produced recommendations by analyzing the relations among the favourite and suggested artists. This is a fundamental feature of a good music recommender system, that should be transparent, i.e. it should be able to provide a convincing explanation of recommendations. Finally, DBpedia might also help to face the other typical problem of content-based filtering approaches, such as the overspecialization [15]. Indeed, suggested artists might be relevant but too similar to those the user likes. This means that suggestions might be accurate but obvious, thus not useful. Hence, we decided to further investigate on the use of DBPedia and Linked Data in order to exploit the wealth of data and relations to obtain more serendipitous (unexpected) results. This allows to focus on some other important aspects besides accuracy for obtaining valuable music recommendations, as devised in a very recent work [20]. 


\section{Conclusions and Future Work}

In this work we presented a first version of MyMusic, a system for building personalized music playlists based on social media sources. This work is based on the idea that information extracted from social networks such as Facebook and Last.fm is very meaningful and can be exploited for personalization tasks. We developed a prototype application able to model user preferences in music and to generate personalized playlists. Moreover, we compared two different techniques for enriching the playlist with new artists related to those the user already likes, the first based on DBpedia and the second based on similarity calculations in vector spaces. In the experimental session we asked users to use the system in order to understand which enrichment technique gained the best results: VSMbased approach was the preferred one. Even if this version of the system is only a simple prototype, results emerged from this preliminary evaluation encouraged keeping on this research. In general, there is still space for future work: first, a deeper evaluation with more users and different values of the parameter $\alpha$ might be useful to evaluate the effectiveness of the systems in building playlists totally based on new singers. Next, the enrichment approaches might be extended by analyzing, respectively, different DBpedia properties or different processing techniques for tags in order to avoid the typical problems of synctatical-based representation. Finally, regarding the extraction step, it might be interesting to introduce the analysis of implicit data extracted from Facebook such as attended events, links, groups and so on.

\section{References}

1. L. Baltrunas, M. Kaminskas, B. Ludwig, O. Moling, F. Ricci, A. Aydin, K. Lüke, and R. Schwaiger. Incarmusic: Context-aware music recommendations in a car. In Christian Huemer and Thomas Setzer, editors, E-Commerce and Web Technologies, volume 85 of Lecture Notes in Business Information Processing, pages 89-100. Springer, 2011.

2. J. Bu, S. Tan, C. Chen, C. Wang, H. Wu, L. Zhang, and X. He. Music recommendation by unified hypergraph: combining social media information and music content. In Proceedings of the international conference on Multimedia, MM '10, pages 391-400, New York, NY, USA, 2010. ACM.

3. O. Celma. Music Recommendation and Discovery - The Long Tail, Long Fail, and Long Play in the Digital Music Space. Springer, 2010.

4. O. Celma and P. Lamere. Music recommendation and discovery revisited. In Bamshad Mobasher, Robin D. Burke, Dietmar Jannach, and Gediminas Adomavicius, editors, Proceedings of the 5th ACM conference on Recommender systems, RecSys '11, pages 7-8. ACM, 2011.

5. O. Celma, M. Ramírez, and P. Herrera. Foafing the Music: A Music Recommendation System based on RSS Feeds and User Preferences. In 6th International Conference on Music Information Retrieval (ISMIR), pages 464-467, London, UK, 2005.

6. O. Celma and X. Serra. FOAFing the Music: Bridging the Semantic Gap in Music Recommendation. Journal of Web Semantics, 6(4):250-256, 2008. 
7. M. J. Eppler and J. Mengis. The concept of information overload: A review of literature from organization science, accounting, marketing, MIS, and related disciplines. Information Society, 20(5):325-344, 2004.

8. S. Golder and B. A. Huberman. The Structure of Collaborative Tagging Systems. Journal of Information Science, 32(2):198-208, 2006.

9. C. Hahn, S. Turlier, T. Liebig, S. Gebhardt, and C. Roelle. Metadata Aggregation for Personalized Music Playlists. HCI in Work and Learning, Life and Leisure, pages $427-442,2010$.

10. J. Ho and R. Tang. Towards an optimal resolution to information overload: an infomediary approach. In Proceedings of the 2001 International ACM SIGGROUP Conference on Supporting Group Work, GROUP '01, pages 91-96, New York, NY, USA, 2001. ACM.

11. G. Kobilarov, C. Bizer, S. Auer, and J. Lehmann. DBpedia - a linked data hub and data source for web applications and enterprises. In Proceedings of Developers Track of 18th International World Wide Web Conference (WWW 2009), April 20th-24th, Madrid, Spain, April 2009.

12. P. Lamere. Social Tagging and Music Information Retrieval. Journal of New Music Research, 37(2):101-114, 2008.

13. Jae Sik Lee and Jin Chun Lee. Context awareness by case-based reasoning in a music recommendation system. In Haruhisa Ichikawa, We-Duke Cho, Ichiro Satoh, and Hee Yong Youn, editors, Ubiquitous Computing Systems, volume 4836 of Lecture Notes in Computer Science, pages 45-58. Springer, 2007.

14. Q. Li, B. M. Kim, D. H. Guan, and D. Whan Oh. A music recommender based on audio features. In Mark Sanderson, Kalervo Jrvelin, James Allan, and Peter Bruza, editors, SIGIR, pages 532-533. ACM, 2004.

15. P. Lops, M. de Gemmis, and G. Semeraro. Content-based recommender systems: State of the art and trends. In Francesco Ricci, Lior Rokach, Bracha Shapira, and Paul B. Kantor, editors, Recommender Systems Handbook, pages 73-105. Springer, 2011.

16. M. D. Mulvenna, S. S. Anand, and A. G. Büchner. Personalization on the net using web mining: introduction. Communications of the ACM, 43(8):122-125, 2000.

17. A. Passant and Y. Raimond. Combining Social Music and Semantic Web for MusicRelated Recommender Systems. In Social Data on the Web, Workshop of the 7th International Semantic Web Conference, Karlsruhe, Deutschland, Oktober 2008.

18. Sasank Reddy and Jeff Mascia. Lifetrak: music in tune with your life. In Proceedings of the 1st ACM international workshop on Human-Centered Multimedia, pages 2534, 2006.

19. G. Salton, A. Wong, and C. S. Yang. A vector space model for automatic indexing. Commun. ACM, 18(11):613-620, 1975.

20. M. Schedl, D. Hauger, and D. Schnitzer. A Model for Serendipitous Music Retrieval. In Proceedings of the 2nd Workshop on Context-Awareness in Retrieval and Recoomendation, 2012.

21. U. Shardanand. Social information filtering for music recommendation. Master's thesis, Massachusetts Institute of Technology, Dept. of Electrical Engineering and Computer Science, 1994.

22. D. Wang, T. Li, and M. Ogihara. Are tags better than audio? the effect of joint use of tags and audio content features for artistic style clustering. In ISMIR, pages $57-62,2010$. 